\title{
Alignment measurement technique for satellite assembly, integration, and test
}

Asmaliza Zulkifli*, Nor Hafizah Abdullah, Ng Su Wai, Wong Soo Mee, Maszlan Ismail

Space System Development and Operational Division, National Space Agency of Malaysia, Selangor, Malaysia

\section{ARTICLE INFO}

Article history:

Received 18 February 2017

Received in revised form

24 July 2017

Accepted 24 July 2017

\section{Keywords:}

Alignment

Orientation measurement

Position measurement

Assembly integration and test

Inspection and verification

\begin{abstract}
A B S T R A C T
In a satellite development cycle, alignment measurement is crucial especially during assembly and integration of satellite part and component as well as a series of mechanical tests performed onto it. It plays a vital role in ensuring critical components of satellite such as antenna, solar panels and reaction wheels are correctly and precisely aligned as per design specifications. Besides, it serves as complimentary analyses for pre and post inspection and verification during mechanical tests. Combination of two measurement parameters, namely the orientation (angle) and the position (distance) will provide complete coordinate knowledge of satellite system. Selection of appropriate measurement technique and equipment on large scale measurement play significant factor in determining the whole measurement plan. Further analyses of measured data will be performed on metrology software, Spatial Analyzer. Details on technique used for satellite alignment measurement are elaborated. A measurement model and real measurement data emphasizing on effectiveness of the mentioned technique are presented.
\end{abstract}

(C) 2017 The Authors. Published by IASE. This is an open access article under the CC BY-NC-ND license (http://creativecommons.org/licenses/by-nc-nd/4.0/).

\section{Introduction}

In recent years, metrology technology has advance steadily with the emergence of a series of reliable high-end metrology instrument particularly on distance and angle measurement. Availability of high-precision instruments has contribute to increase in productivity and efficiency in various fields such as automotive (Bogue, 2008), aerospace (Aviado et al., 2006; Ravishankar et al., 2010; Wang et al., 2015), large-scale assembly (Cuypers et al., 2009; Li et al., 2014; Zheng et al., 2013) and manufacturing (Jaramillo et al., 2011; Su and Rowlands, 2000).

Typically laser system instrument will be used in the distance measurement for its high-accuracy and to shorten measurement time compared to conventional method (Gao et al., 2015). Laser trackers and theodolites have been used intensively in the aerospace industry to assist assembly and integration of large parts of airplane (Wang et al., 2015) or satellite components (Aviado et al., 2006; Ravishankar et al., 2010), inspection after mechanical and environment test for any defect or

\footnotetext{
* Corresponding Author.

Email Address: asmaliza@angkasa.gov.my (A. Zulkifli)

https://doi.org/10.21833/ijaas.2017.09.016

2313-626X/C) 2017 The Authors. Published by IASE.

This is an open access article under the CC BY-NC-ND license

(http://creativecommons.org/licenses/by-nc-nd/4.0/)
}

structural deformation (Bogue, 2008; Jaramillo et al., 2011) and verification of part assembly compare to its reference engineering model (CAD) ( $\mathrm{Su}$ and Rowlands, 2000).

In the satellite development cycle, assembly of parts/components and integration of subsystems requires very tight tolerance in measurement accuracy for both distance and angle that shall be incorporate in the satellite coordinate system. This stringent requirement will ensure parts and subsystems functionality is achieved according to design specifications.

Leica Geosystems and Faro are two reliable manufacturers of measurement products in the global market. Their products have been proven globally and widely used in various fields, including our facility, i.e. Malaysian Satellite Assembly, Integration and Test (AIT) which is located at Malaysia Space Centre, Banting, and Selangor. The AIT facility is equipped with 6 testing and measurement equipment namely Vibration Test System (VTS), Reverberation Acoustic Test Facility (RATF), Mass Properties Measurement System (MPMS), Electromagnetic Compatibility Chamber (EMC), Thermal Vacuum Chamber (TVC) and Alignment Measurement System (AMS) with capability of performing mechanical and environmental tests for any test article below $1000 \mathrm{~kg}$. 


\section{Measurement framework}

With presence of advance metrology instruments, alignment measurement of large part or assembly becomes easier. Typically at any satellite assembly, integration and test facility, combination use of laser trackers and theodolites is adopted to cater both position and orientation measurement of the test article as shown in Fig. 1 (Kress et al., 1998).
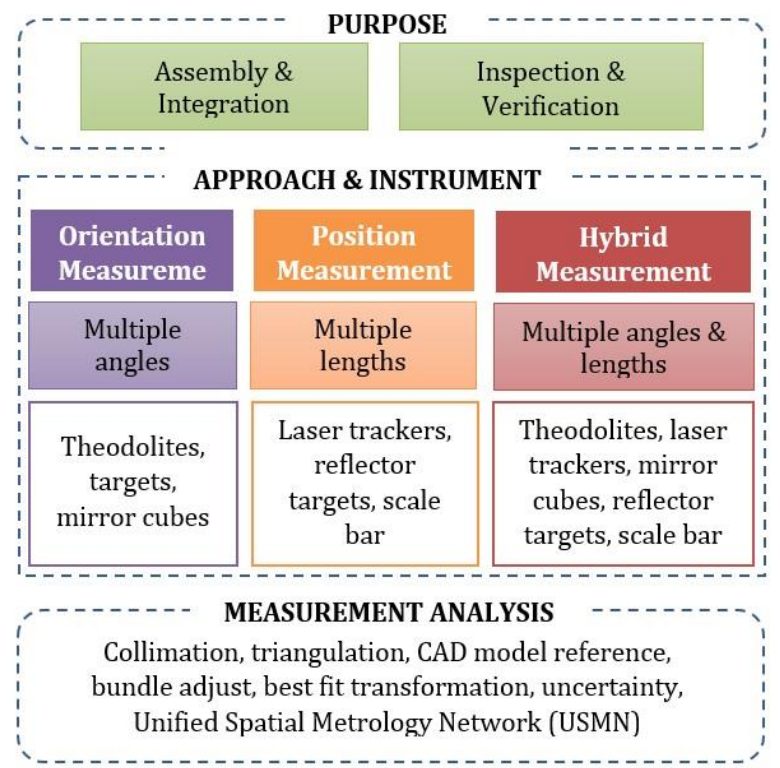

Fig. 1: Measurement framework

This combination is able to increase accuracy (Dubock et al., 2001), efficiency (Wu and Su, 2015), reduce cost and shorten measurement time (Ravishankar et al., 2010) during assembly and integration of parts/subsystems and inspection and verification activity. Both instruments are laboratory friendly and comply with the laboratory environmental requirement.

Automotive industry especially car production use a laser-based geometrical inspection technique (Bogue, 2008; Muralikrishnan et al., 2016) to speed up inspection activity while maintaining high accuracy in delivering a high quality product. Comparison of reference model (i.e. CAD model, premeasured data) with actual measurement data by using best fit transformation technique (Forbes et al., 2009) ensure manufactured product met its specification.

From satellite development perspective, alignment measurement is required during assembly and integration of parts and subsystems and inspection and verification before and after a series of mechanical and environmental test. Generally, principle of measurement can be categorized into 3 approaches, i.e. orientation (angular), position (distance) and hybrid (combination of both orientation and position measurement) (Cuypers et al., 2009; Li et al., 2014) as per Fig. 1. Selection of a measurement approach depends on requirement which in turn will determine tools and analysis techniques.
Orientation measurement approach provides best accuracy in terms of angle information, i.e. 0.5 arcsec (Leica; 2016). The advantages include capability in large working volume, high resolution measurement and immune to electromagnetic interferences (Li et al., 2014). On multiple angles measurement, more than 2 theodolites are required to aim at each other mutually and collimate with the mirror cubes on the measured article. Such requirement results in uncertainty arise due to operator handling which contribute to low efficiency (Li et al., 2014).

Position measurement approach provides distance accuracy from $15 \mu \mathrm{m}$ to $0.5 \mathrm{~mm}+6-13 \mu \mathrm{m} / \mathrm{m}$ (Leica, 2016) depends on type of laser tracker and its reflector targets. Fig. 2 show example of metrology products for orientation and position measurement.
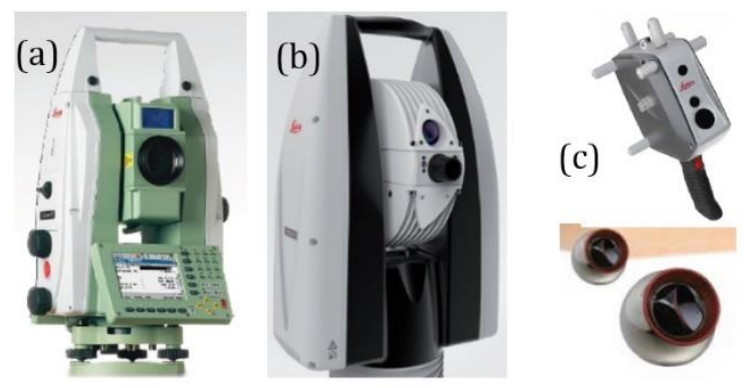

Fig. 2: (a) Theodolite TM6100A (b) Laser tracker ATR960 (c) T-scan, mirror cube, reflector and target kit

\section{Assembly and integration}

During development of a satellite, reference mirror cube will be installed on satellite bus mainframe as a reference datum for parts assembly and subsystems integration. Parts such as solar panels, thrusters, reaction wheels and attitude determination sensors will be assemble, integrate and align to the reference mirror as shown in Fig. 3.

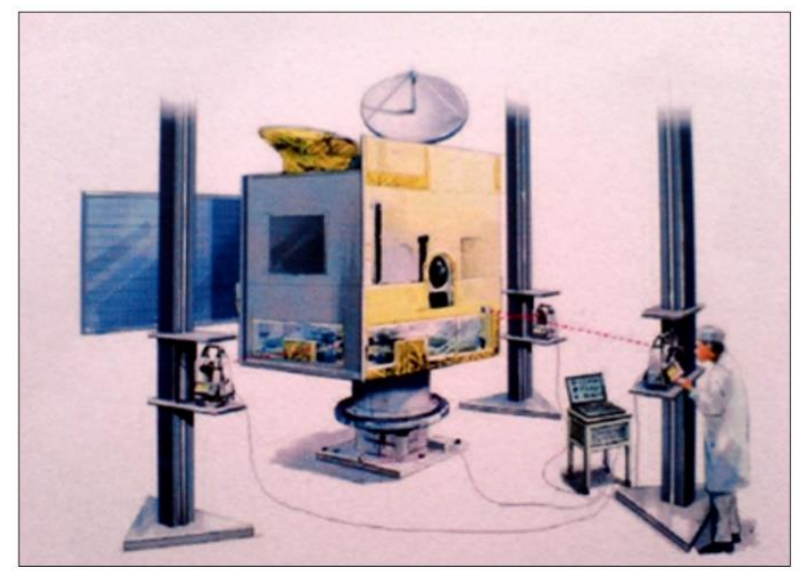

Fig. 3: Illustration of satellite alignment activity

For example, remote sensing satellites which carry an electro-optical camera require a 3-axis stabilization during its mission operation. The stabilization can be achieve using attitude actuators such as reaction wheels, momentum wheels, 
propulsion system and others type of actuators with knowledge of attitude sensors (star tracker, sun sensors, gyro, magnetometer, etc.). Therefore, it require meticulous works and tremendous efforts in ensuring placement and alignment of assemble parts are accurate and precise as per satellite design specification.

Fig. 4 shows a RazakSAT components placement configuration as well as external view of stowed configuration.
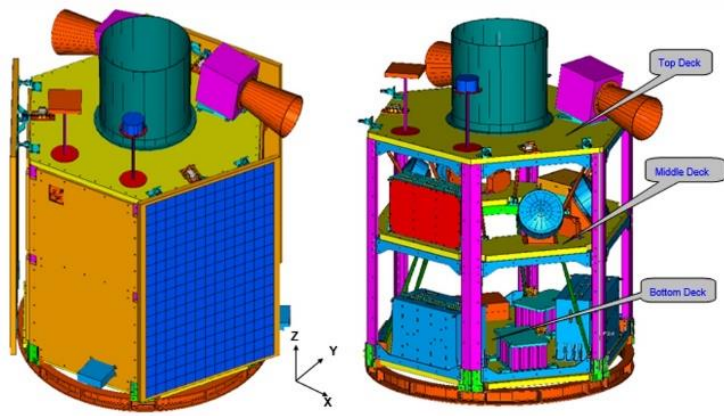

Fig. 4: RazakSAT component placement
With regards to the development of a geostationary communication satellite, assembly and integration activities is conducted on both phases; pre-environment tests and post-environment tests (Ebadi, 2013) as per Fig. 5. In pre-environment tests phase, various parts and components of a particular subsystem are assembled and integrated to build a subsystem module (i.e. payload, ADCS, communication) prior to subsystems integration to satellite bus mainframe structure. This includes integration of antenna and solar panels before mechanical and environmental tests phase.

For post-environment tests phase, satellite subsystems operability and functionality will be verified after a series of mechanical and environmental tests. This includes conducting final integration system test (FIST) and final alignment survey (Ebadi, 2013) before proceed to the launch site. Solar panel wings and antenna transponders deployment tests shall be conduct using special mechanical ground support equipment (MGSE) to simulate zero gravity environment (Kress et al., 1998).

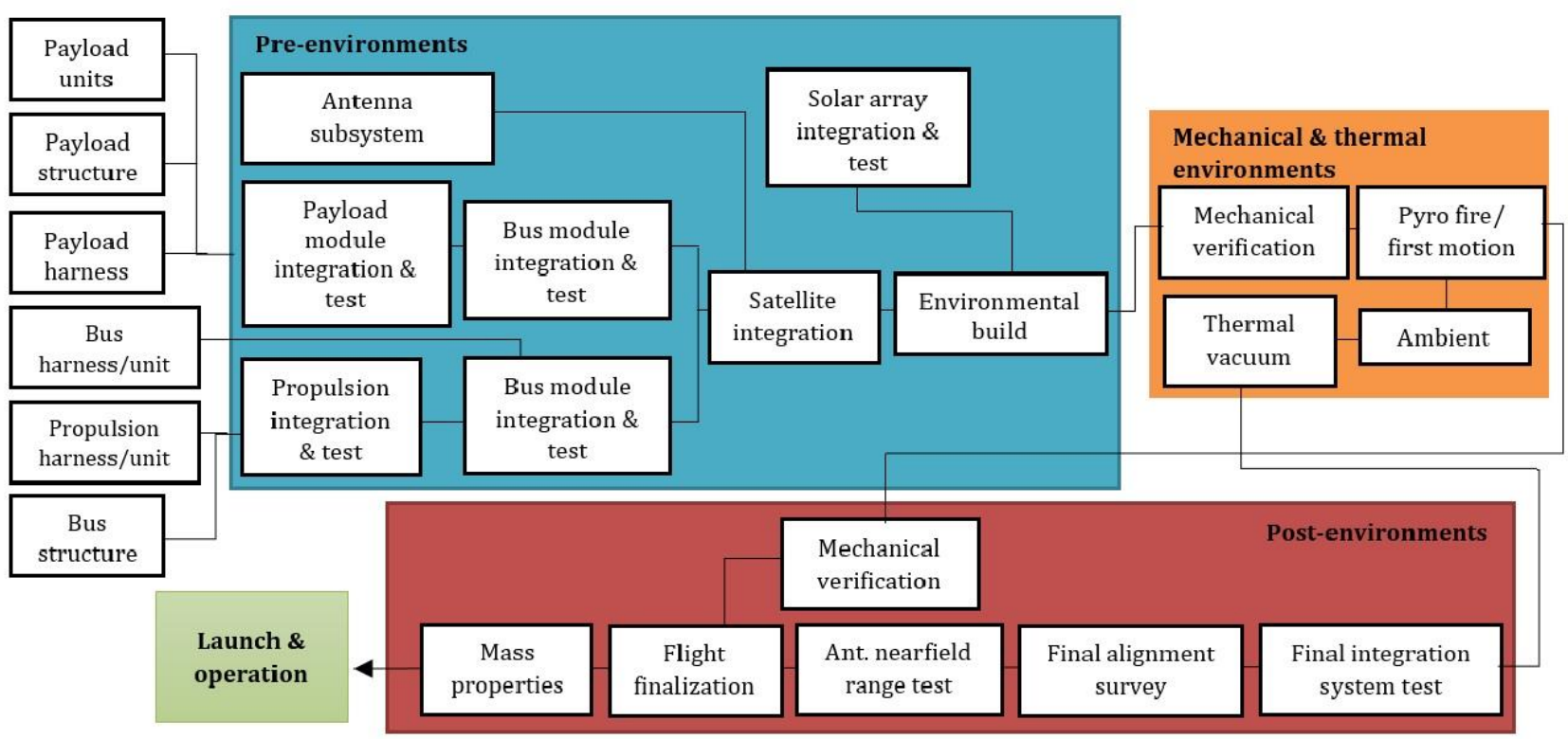

Fig. 5: Assembly, integration and test flow for a geostationary communication satellite (Ebadi, 2013)

Prior to measurement, a Cartesian coordinate system shall be established according to reference mirror and assembly and integration of parts/subsystems shall be precisely aligned to it. Fig. 6 shows measurement model for orientation measurement and hybrid measurement approaches.

A reference theodolite shall installed into fixed location and collimate with other theodolites used in the measurement. Theodolites shall collimate itself with mirror cube and other theodolite to complete a triangle. Mirror measurements are presented with reference to theodolite's azimuth (theta) and elevation (phi) information. This information will be used to calculate mirror collimation properties as per following formula (Eqs. 1-3):

$\theta_{\mathrm{a}}=\theta_{\mathrm{coLA}}-\theta_{\mathrm{mf} 1}$

$\theta_{\mathrm{b}}=\theta_{\mathrm{coLB}}-\theta_{\mathrm{mf} 2}$ $\theta_{\mathrm{a}}+\theta_{\mathrm{b}}+\theta_{\text {mirror }}=0$

Hybrid measurement will require a scale bar (or a laser tracker) measurement for distance information. These data can be recorded directly into Spatial Analyzer or flash memory for analysis.

\section{Inspection and verification}

Development cycle of a satellite consists of a series of mechanical and environmental tests as explain in Fig. 5. It will demonstrate satellite subsystems functionality under harsh environment including exposure to extreme temperature, vacuum environment, shock and acoustic vibration (Dubock et al., 2001; Ebadi, 2013). Simulation of a real environment during launching and in orbit operation 
will be conducted to validate performance with respects to the operational specifications.

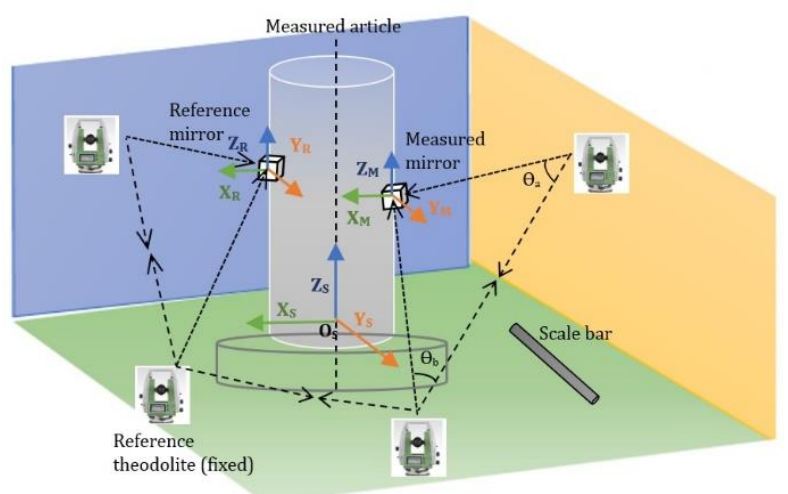

Fig. 6: Measurement model

A geostationary satellite require at least two (2) cycles of environment test and a cycle of mechanical test as depicted on Fig. 5. In pre-environment phase, a series of test will be conducted onto parts and subsystems level. While in post-environment phase, a complete integrated system (a complete satellite) will undergo a series of test. It is important to establish a pre-environmental reference by verifying and validating all functional requirements for all parts and subsystems before integration is conducted.

During post-environment test, final alignment survey and inspection are conducted especially on satellite's solar panel deployment, attitude actuators and sensors to verify and validate the previous measurement.

Prior to any mechanical and environmental test, a satellite model will be measured using laser trackers (position measurement) with pre-defined target points installed on satellite surface using special adhesive. Group of target points will be measured and recorded as a reference points. Position measurement approach is suitable to be used in this activity to speed up the measurement time since it involves a lot of target point measurement.

The same pre-defined target points will be measured again after the tests. Comparison of reference and actual data will be performed by using metrology software, e.g. Spatial Analyzer. For inspection and verification activity, best fit transformation technique is most suitable to visualize deviation information on each axis $(x, y, z)$ between reference and actual target points. Detail analysis of structural deformation information of a measured article can be obtained from error (deviation) magnitude.

Table 1: Measurement data (position measurement)

\begin{tabular}{|c|c|c|c|c|c|c|c|c|c|c|}
\hline $\begin{array}{c}\text { Target } \\
\text { Point }\end{array}$ & RefX & Ref Y & Ref Z & Act X & Act $Y$ & Act Z & $\mathrm{dX}$ & $d Y$ & $\mathrm{dZ}$ & dMag \\
\hline M1 & -6895.89 & 3188.192 & -1447.88 & -6896.76 & 3188.013 & -1447.89 & -0.8749 & -0.1785 & -0.0102 & 0.893 \\
\hline M2 & -3322.74 & 3031.84 & -1444.92 & -3323.6 & 3031.887 & -1444.91 & -0.8611 & 0.0473 & 0.0043 & 0.8624 \\
\hline M3 & -4225.98 & 934.4474 & -1424.74 & -4221.67 & 934.4029 & -1424.73 & 4.312 & -0.0444 & 0.0068 & 4.3122 \\
\hline M4 & -2544.19 & 221.591 & -1417.88 & -2544.9 & 221.6079 & -1417.87 & -0.7092 & 0.0169 & 0.0057 & 0.7094 \\
\hline M5 & -4011.06 & -846.131 & -1412.62 & -4011.7 & -846.171 & -1412.61 & -0.6463 & -0.0408 & 0.0079 & 0.6476 \\
\hline M6 & -2392.57 & -1981.64 & -1402.03 & -2393.15 & -1981.61 & -1402.04 & -0.5892 & 0.0375 & -0.0056 & 0.5904 \\
\hline M7 & -471.238 & -1630.45 & -1404.61 & -471.869 & -1630.29 & -1404.62 & -0.6313 & 0.1619 & -0.0089 & 0.6518 \\
\hline
\end{tabular}

\section{Measurement analysis}

Prior to measurements, connection between acquisition computer and equipment/instrument shall be established via cable or Bluetooth for faster data processing and analysis. In this measurement, Spatial Analyzer metrology software from New River Kinematics will be used. We have conducted position (distance) measurement by using a laser tracker with 7 target points (M1, M2, ..., M7) before and after a mechanical test (vibration).

Measured data is recorded into group points (before::Ref) and (after::Act) as shown in Table 1. Best fit transformation technique has been applied on measurement data to analyze deviation of structural properties on target points. Analysis results are shown in Table 2 and Fig. 7.

Table 2 shows that $\mathrm{x}$-axis measurements contribute highest percentage of error with deviation about $4.3 \mathrm{~mm}$. Cross analysis with Fig. 7 shows that point M3 is major contributor for measurement error. From the result, we could derive an assumption that point M3 has weakest structural properties which resulting a structural deformation.

Table 2: Best fit transformation result

\begin{tabular}{ccccc}
\hline Results & $\mathrm{X}$ & $\mathrm{Y}$ & $\mathrm{Z}$ & Mag. \\
\hline Count & 7 & 7 & 7 & 7 \\
Max Error & 4.312 & 0.1785 & 0.0102 & 4.3122 \\
RMS Error & 1.7634 & 0.0968 & 0.0073 & 1.7661 \\
StdDev Error & 1.9047 & 0.1046 & 0.0079 & 1.9076 \\
\hline
\end{tabular}

\section{Unified spatial metrology network}

Unified Spatial Metrology Network (USMN) is an advanced tool for combining common measurements for two or more instruments into a single and ideal instrument network. It can calculate uncertainty by weighted bundle concept and provide mean to real-world and unrealistic uncertainty on an individual basis. Besides that, it also leverages the uncertainty characteristics of different instruments to provide a much more accurate instrument network than that of traditional alignment methods. 


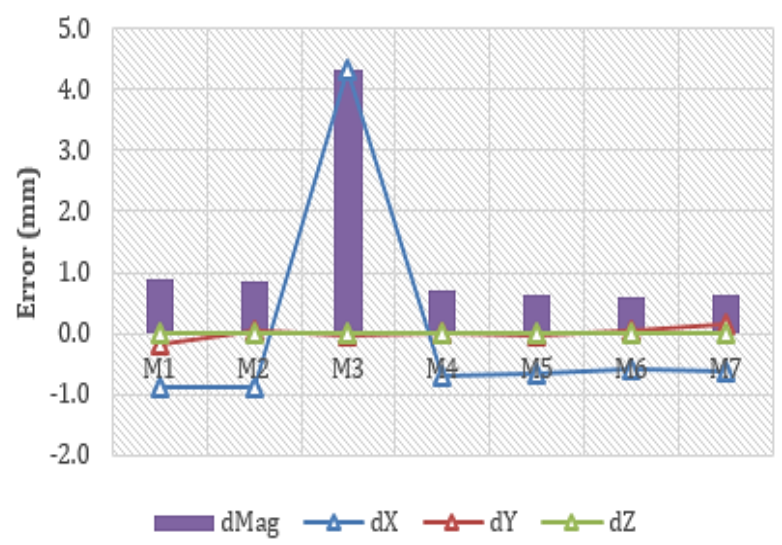

Fig. 7: Best fit transformation error

On large-scale measurement where multiple type of instruments involved, bundle adjust or best fit transformation technique itself could not provide full and comprehensive analysis offered by USMN.

For example, on multi instruments measurement where four (4) laser trackers involved, a USMN composite point will be generated by considering all possible uncertainties (measurement, instruments, environment, etc.). The composite point is representing the ideal position of each point of measurement and has uncertainty properties for further analysis.

Fig. 8 shows a generated USMN composite point locate nearer to Tracker 3 and 4 points compare to other points as depicted in Table 3. From the Fig. 8 and Table 3 , we can clearly identify Tracker 1 as major contributor to the highest uncertainty value compared to other trackers (highest relative magnitude).

Table 3: M1 coordinates

\begin{tabular}{lcccc}
\multicolumn{5}{c}{ Table 3: } \\
\hline Point: M1 & $\mathrm{x}$ & $\mathrm{y}$ & $\mathrm{z}$ & $\mathrm{mag}$ \\
\hline Tracker 1 & -1904.017 & -3934.257 & 887.356 & 0.0689 \\
Tracker 2 & -1904.029 & -3934.169 & 887.41 & 0.0375 \\
Tracker 3 & -1904.033 & -3934.201 & 887.388 & 0.0187 \\
Tracker 4 & -1904.003 & -3934.206 & 887.369 & 0.0246 \\
USMN & -1904.015 & -3934.196 & 887.388 & \\
\hline
\end{tabular}

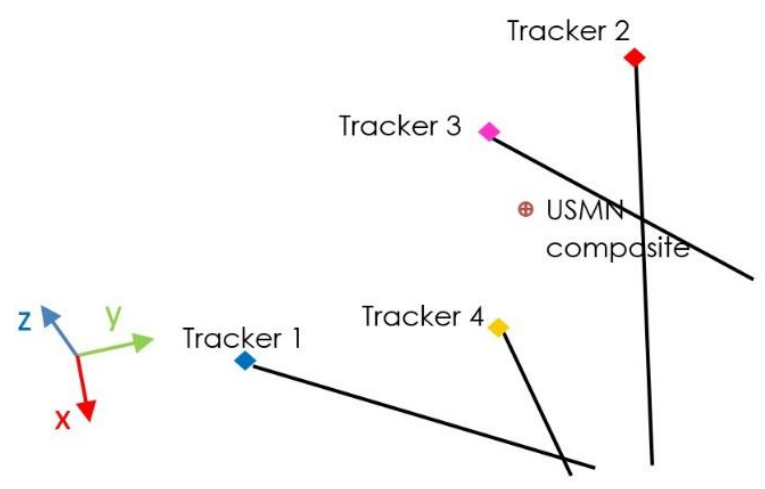

Fig. 8: USMN composite point

\section{Conclusion}

Alignment measurement plays vital roles in satellite development especially during assembly and integration of parts and subsystems by using combination of orientation and position measurement. Measurement requirement shall be specified and the appropriate selection of approach, instruments and analysis technique will yield best result. Best fit transformation and USMN technique are used to provide detail analysis of measurement.

\section{References}

Aviado C, James G, and Kevin R (2006). Methods for correlating autocollimation of theodolites and coordinate metrology in spacecraft systems. In the Conference of SPIE Astronomical Telescopes Instrumentation on Optomechanical Technologies for Astronomy, International Society for Optics and Photonics Orlando, USA: 62733H-62733H. https://doi.org/10.1117/12. 670043

Bogue R (2008). Car manufacturer uses novel laser scanner to reduce time to production. Assembly Automation, 28(2): 113114.

Cuypers W, Van Gestel N, Voet A, Kruth JP, Mingneau J, and Bleys P (2009). Optical measurement techniques for mobile and largescale dimensional metrology. Optics and Lasers in Engineering, 47(3): 292-300.

Dubock PA, Spoto F, Simpson J, Spencer D, Schutte E, and Sontag H (2001). The envisat satellite and its integration. ESA Bulletin, 106: 26-45.

Ebadi AR (2013). Communication satellite fundamentals, from design to launch and operation. Oxford University Press, Oxford, UK.

Forbes AB, Hughes B, and Sun W (2009). Comparison of measurements in co-ordinate metrology. Measurement, 42(10): 1473-1477.

Gao W, Kim SW, Bosse H, Haitjema H, Chen YL, Lu XD, Knapp W, Weckenmann A, Estler WT, and Kunzmann H (2015). Measurement technologies for precision positioning. CIRP Annals-Manufacturing Technology, 64(2): 773-796.

Jaramillo AE, Boulanger P, and Preito F (2011). On-line 3-D system for the inspection of deformable parts. International Journal of Advanced Manufacturing Technology, 57(9-12): 1053-1063.

Kress H, Habersack J, and Paus S (1998). Alignment concept for spacecraft antennas in state of the art test facilities. In Proceedings of AMTA, Montreal, Canada: 98: 358-362.

Leica (2016). Product Brochures - Absolute Tracker AT960, Laser Station TDRA6000 and Industrial Theodolite TM6100A. Leica Geosystems Surveying Company, St. Gallen, Switzerland.

Li Y, Qiu Y, Chen Y, and Guan K (2014). A novel orientation and position measuring system for large and medium scale precision assembly. Optics and Lasers in Engineering, 62: 3137.

Muralikrishnan B, Phillips S, and Sawyer D (2016). Laser trackers for large-scale dimensional metrology: A review. Precision Engineering, 44: 13-28.

Ravishankar S, Dutt H, and Gurumoorthy B (2010). Automated inspection of aircraft parts using a modified ICP algorithm. The International Journal of Advanced Manufacturing Technology, 46(1-4): 227-236.

Su Z and Rowlands H (2000). A laser alignment system for boat assembly. Sensor Review, 20(3): 206-211.

Wang Q, Huag P, Li J, and Ke Y (2015). Boresighting method of the aircraft gun using a laser tracker. Sensor Review, 35(3): 251262.

Wu B and Su X (2015). A novel precise guiding method for visual guiding theodolite measurement in volume space. OptikInternational Journal for Light and Electron Optics, 126(23): 3969-3973. 
Zheng L, Zhu X, Liu R, Wang Y, and Maropoulus PG (2013). A novel algorithm of posture best fit based on key characteristics for large component assembly. Procedia CIRP, 10: 162-168. 